\title{
An alternative, spiritual and new age interpretation of Schizophrenia
}

Beyer AC

Beyer AC. An alternative, spiritual and new age interpretation of Schizophrenia. Curr Res Integr Med 2017;3(S1):1.

\section{EDITORIAL}

This paper is an attempt to interpret the illness of schizophrenia from a new viewpoint. It starts with a background that shows the importance of the spiritual perspective at the history of the illness. It goes on to present an interpretation of the positive and negative symptoms generally in schizophrenia and the common conditions of developing it. It then presents an overview over some symptoms. It connects some of the symptoms to the spiritual or new age literature, where these are known occurrences without necessarily a diagnostic impact. It then presents some additional common 'symptoms' that have not yet been explained in this literature. It concludes with some general recommendations for either treatment or self-help.

\section{BACKGROUND}

Schizophrenia increased since about 1750. That was about the time when secularisation replaced a spiritual outlook on life, and many of the commonly problematic stimulants connected to schizophrenia (in particular coffee and nicotine) were introduced. It would hence be logical to search in these factors at least some of the causes of the illness, which today affects about 52 million worldwide, men and women alike, usually starting in early adulthood, with often severe costs for themselves and society. An additional factor that could potentially be related to this is the very high apparent presence of the illness in China, an atheist country. At this period in history, it seems also that culturally homogenous areas (in Britain) and countries (China versus India) experience a higher frequency of this illness. This is logical if one accepts the interpretation below, as a restriction of spirituality or fear of cultural exchange might create paranoia in culturally homogenous areas more than in mixed cultures. These, of course, are just some potential factors, and under different social and political conditions it could also be the other way around, depending on the presence of conflict or its absence.

\section{SYMPTOMS IN SCHIZOPHRENIA}

Positive and negative symptoms: positive are delusions, voices, etc., negative are apathy, lack of speech etc. The focus will here be on positive symptoms. Negative symptoms are here thought to be a reaction to the positive symptoms (being afraid, stunned, confused, not being able to talk about them because they are so weird) and a rejection to the often resulting stigma, social isolation and often ridicule (basically resembling depression). An additional interpretation, also used here but named benign or not benign symptoms refers to the quality of positive symptoms, which means perceptions that are uncommon to the Western mind and can have either a wanted and pleasant (benign) or unpleasant and unwanted (not benign) quality.

Preconditions (isolation due to migration or discrimination, trauma (from deaths in the family to sudden unemployment to political negative events, such as wars etc.), drugs, (self)-starvation or lack of sleep/exhaustion, other negative life conditions or events, withdrawal, sometimes asocial behaviour etc.). These are commonly known to potentially precede spiritual 'interventions' and hence the first episode of psychosis.

\section{SYMPTOMS THAT RESEMBLE NEW AGE AND SPIRITUAL PHENOMENA}

Visions - visionary illusions - could be clairvoyance or traditional 'visions'.

Auditory hallucinations ('voices') - could be telepathy amongst humans, communications from beings from other dimensions (angels, spirits, demons) or alien communication (it has been revealed by a former CIA member of staff that aliens might communicate telepathically).

Aspects that occur that are also present in the new age spiritual literature and are known in other cultures, such as Islam and Buddhism: Seeing auras and energies, seeing orbs and or light flashes, out of body experiences, precognition and precognitive or telepathic dreams, intensified occurrence of astounding or banal synchronicities (a common occurrence apparently, from numbers to strange cloud formations, many different things), complete hallucinatory journeys similar to the shamanic journey or possibly to visions of saints, near death experiences (NDEs), bodily effects such as stigmata or wounds that have no known origin.

Strange ideas with various content (from science to religion to artistic or social ideas etc.).

\section{OTHER SYMPTOMS NOT YET IDENTIFIED IN THE SPIRITUAL OR NEW AGE LITERATURE}

Thought broadcasting (feeling that one's personal thoughts are 'readable' or 'audible' to many people, this does not mean written or spoken thoughts, but literally thoughts in the head only).

Personal communication received for example via the TV.

The common fact that many (but by far no all!) of the symptoms are presenting with negative, aggressive content (which is the most common form of symptoms in paranoid schizophrenia, which is most common diagnosis).

Paranoia and (not necessarily always identical with the former) a common belief in being pursued by the intelligence services etc. Could possibly be explained either by a general 'primordial' fear of Westerners of the CIA etc. or indeed by the fact that the security state watches paranoid schizophrenics (compare the security and surveillance state in Britain since 2008 approx.). The same has been reported from other world regions and in relation to different political entities.

Also 'parapsychological warfare' - perceptions of being physically attacked by immaterial entities with potential physical or psychosomatic effects.

Other unexplained phenomena, such as visions of unknown creatures that seem to have a fantastical quality, or spiritual entities or phenomena from all sorts of cultures, hauntings, such as feelings of insects crawling the skin etc. (which are sometimes reported by some patients).

The explanation for these could simply be the presence of conflicts, changes or challenges. In the presence of any conflicts or trauma, such as anything from dysfunctional families, to war, to discrimination, to unemployment etc., parapsychological perceptions will naturally turn negative and haunting. This then is called 'paranoid schizophrenia'. Oftentimes, if the patient attempts to find a positive solution, these not benign symptoms might turn into benign symptoms or simply recede again, similar to other life challenges. Change of content of symptoms is as common in schizophrenia as change in experiences in non-schizophrenic lives and depends on similar factors.

\section{RECOMMENDATIONS FOR TREATMENT}

Spiritual literatures have long standing tradition of recommendations. Spiritual discipline and becoming a happier, more benign and loving person, less fearful and more independent also, not necessarily sticking to

Faculty of Buisness, Law and Politics, University of Hull, United Kingdom

Correspondence: Beyer AC, Faculty of Buisness, Law and Politics, University of Hull, United Kingdom, Telephone: +07554193888, e-mailto:C.Beyer@hull.ac.uk

Received: November 6, 2017, Accepted: November 9, 2017, Published: November 20, 2017

This open-access article is distributed under the terms of the Creative Commons Attribution Non-Commercial License (CC BY-NC) (http:// OPEN ACCESS creativecommons.org/licenses/by-nc/4.0/), which permits reuse, distribution and reproduction of the article, provided that the original work is properly cited and the reuse is restricted to noncommercial purposes. For commercial reuse, contact reprints@pulsus.com 
dogma, but still becoming spiritual in a broad sense, including adopting vegetarianism, meditation, general ethics, avoidance of drugs, an open mind accepting the unknown, reading of regarding literature to familiarise oneself with these concepts, adopting a variety of spiritual practices - from mantras, to meditation, to physical exercises such as tai chi, to prayer, to various alternative healing practices etc. According to taste or need, seem helpful. In addition, supplementation with vitamins (omega 3, niacin, vitamin C) can aid mental and physical strength and healing and reduction in stimulants (coffee, alcohol, nicotine, drugs) seems helpful to avoid escalation of symptoms, such as paranoia and voices in particular. However, a too strict health discipline can also create depression. Healthy living in general is often a problem if the main reliance for treatment is on neuroleptics. Many schizophrenics die early of either suicide or other common health problems, which can be prevented with a better health discipline. Neuroleptics can help in emergencies, but lead nowhere if not additional measures are taken (medicine admits that the neuroleptics cannot heal schizophrenia, just suppress the symptoms, with often accompanying severe side effects). Some studies even claim that most people that recover completely used either little or no neuroleptics, and this has at least partially been confirmed in a small informal online survey on Facebook conducted in 2017 to which 18 schizophrenia patients and former patients responded. The results of this survey indicated that spirituality (unclassified, Buddhism, Christianity and Islam seemed likewise helpful, shamanism seemed not quite as helpful) and a more than standard healthy diet (including fasting, veganism, supplementation with vitamins such as Omega 3) including moderate physical exercise were a a potential winning combination long term and had been used by those who had recovered for many years. Lifestyle factors - from living conditions to education and employment to entertainment to social relations - seem also important for recovery, according to the literature and this survey. Medication still can be very useful for handling emergencies or for example paranoia when these approaches mentioned here take longer to show results. But they should be minimised, as far as possible, and reduced in duration likewise. Other coping strategies, life and social skills are at least as important and need to be acquired to cope long term. On the other hand, what tools and methods might work at any time might might possibly differ from patient to patient and from situation to situation. 